\title{
SEPARATING INTRINSIC AND MICROLENSING VARIABILITY USING PARALLAX MEASUREMENTS
}

\author{
STEIN VIDAR HAGFORS HAUGAN \\ Institute of Theoretical Astrophysics, University of Oslo \\ Pb. 1029, Blindern \\ N-0315 OSLO \\ http://www.uio.no/ ^steinhh/index.html
}

\section{Introduction}

In gravitational lens systems with 3 or more resolved images of a quasar, the intrinsic variability may be unambiguously separated from the microlensing variability through parallax measurements from 3 observers when there is no relative motion of the lens masses (Refsdal, 1993). In systems with fewer than 3 resolved images, however, this separation is not straightforward. For the purpose of illustration, I make the following simplifications for the one-dimensional case: The observations consist of well-sampled time series of the observed flux $F_{\mathbf{A}}\left(t_{i}\right)$ and $F_{\mathbf{B}}\left(t_{i}\right)$ at two points in the observer plane. The separation vector of the two points is parallel to the direction of the transversal motion of the source-lens-observer system, and the distance $D_{\mathrm{AB}}$ between the observers is known. Furthermore, the distance $D_{\mathrm{AB}}$ is small compared to the typical length scale of fluctuations in the magnification $\mu(x)$.

It is possible to calculate the ratio of the instantaneous magnification at the two observers as a function of time, defined by

$$
r\left(t_{i}\right)=F_{\mathbf{B}}\left(t_{i}\right) / F_{\mathbf{A}}\left(t_{i}\right)
$$

where $F_{\mathbf{A}}\left(t_{i}\right)$ and $F_{\mathbf{B}}\left(t_{i}\right)$ are the observed fluxes at observer $\mathbf{A}$ and $\mathbf{B}$ respectively. I am assuming that observer $\mathbf{B}$ is the leading one.

With these assumptions, the magnification history $\mu_{\mathbf{A}}\left(t_{i}\right)$ for observer A, can be reconstructed (apart from boundary conditions) through the formula

$$
\mu_{\mathbf{A}}\left(t_{i}\right)=\mu_{\mathbf{A}}\left(t_{i}-\Delta t\right) r\left(t_{i}-\Delta t\right) \quad \text { with } \quad \Delta t=\frac{D_{\mathbf{A B}}}{v_{\perp}}
$$


where $v_{\perp}$ is the unknown velocity perpendicular to the line of sight.

Given a velocity $v_{\perp}$, the microlensing magnification history $\mu_{\mathbf{A}}$ is uniquely determined, and thereby also the intrinsic flux, given by

$$
F_{\mathbf{I A}}\left(t_{i}\right)=F_{\mathbf{A}}\left(t_{i}\right) / \mu_{\mathbf{A}}\left(t_{i}\right)
$$

The velocity is chosen by minimizing some measure of the variability (e.g., $\left.\chi^{2}\right)$ of $F_{\mathbf{I A}}$, given by $\chi^{2}=\sum_{i=1}^{N}\left(F_{\mathbf{I A}}\left(t_{i}\right)-\left\langle F_{\mathbf{I A}}\right\rangle\right)^{2}$

\section{Preliminary results}

In order to test the method, dummy data for the intrinsic flux $F_{\mathbf{I}}\left(t_{i}\right)$ and the magnification $\mu\left(x_{i}\right)=\mu\left(v_{\perp} t_{i}\right)$ were made by simply filtering white noise, $N(t)$, with gaussian low-pass filters with characteristic scales $\tau_{\mathbf{I}}$ and $\tau_{\mu}$, and then exponentiating, e.g.:

$$
\begin{aligned}
& F_{\mathbf{I}}(t)=\exp \left(A_{\mathbf{I}} \Phi\left[N(t) ; \tau_{\mathbf{I}}\right]\right) \\
& \mu(t)=\exp \left(A_{\mu} \Phi\left[N(t) ; \tau_{\mu}\right]\right)
\end{aligned}
$$

where $\Phi[\ldots ; \tau]$ denotes gaussian filtering with time scale $\tau$, and then renormalization to make the variance equal to one. $A_{\mathbf{I}}$ and $A_{\mu}$ are the amplitudes of the intrinsic and microlensing variabilities, respectively. For simplicity, but without loss of generality, the units were chosen so that the "true" source-lens-observer transversal velocity $v_{\perp}$ and the characteristic scale of the magnification fluctuations $\tau_{\mathbf{I}}$ were equal to 1 . The observations were simulated according to

$$
\begin{aligned}
& F_{\mathbf{A}}\left(t_{i}\right)=F_{\mathbf{I}}\left(t_{i}\right) \mu\left(v_{\perp} t_{i}\right) \\
& F_{\mathbf{B}}\left(t_{i}\right)=F_{\mathbf{I}}\left(t_{i}\right) \mu\left(v_{\perp} t_{i}+\frac{D_{\mathbf{A B}}}{v_{\perp}}\right)
\end{aligned}
$$

The flux ratio $r\left(t_{i}\right)$, the magnification history $\mu_{\mathbf{A}}\left(t_{i}\right)$ and the intrinsic flux $F_{\mathbf{I A}}\left(t_{i}\right)$ were calculated for a range of values for $v_{\perp}$. For a wide range of parameters, the $\chi^{2}$ function is fairly well-behaved, with a quadratic minimum, although the minimum may be somewhat displaced compared to the true value of $v_{\perp}$. The most difficult cases seem to be those where $\tau_{\mathbf{I}} \approx \tau_{\mu}$ and $A_{\mathbf{I}} \gtrsim A_{\mu}$.

It is unclear how useful this method is for the two-dimensional case with two observers. This will be the subject of further study. The extension of the method to 3 observers in two dimensions with is fairly straightforward. In cases where relative motion of the lensing point masses are important, only a partial separation will be possible.

\section{References}

Refsdal, S. 1993, in Gravitational Lenses in the Universe, eds. Surdej et al., Université de Liège, Belgium 